\title{
A versatile, stimulus-responsive nanoparticle-based platform for use in both sonodynamic and photodynamic cancer therapy
}

\author{
N. Nomikou, ${ }^{\mathrm{a}^{*}}$ K.Curtis, ${ }^{\mathrm{b}}$ C. McEwan, ${ }^{\mathrm{b}}$ B.M.G. O’Hagan, ${ }^{\mathrm{b}}$ B. Callan, ${ }^{\mathrm{b}}$ J.F. Callan ${ }^{\mathrm{b} *}$ and A. \\ P. McHale b* $^{*}$
}

a. Division of Surgery and Interventional Sci., University College London, Royal Free Hospital, Pond St., London, NW3 2PF, UK.

b. Biomedical Sciences Research Institute, University of Ulster, Cromore Rd., Coleraine, Co. Derry, BT52 1SA, UK

* Corresponding authors: ap.mchale@ulster.ac.uk (Phone: +42870 124616; FAX: +442870 12318; j.callan@ulster.ac.uk; and n.nomikou@ucl.ac.uk

\begin{abstract}
A PLGA-based multifunctional biodegradable nanoparticle platform co-harboring hematoporporphyrin and indocyanine green has been developed. In vitro studies demonstrate ultrasound and light stimulated generation of cytotoxic reactive oxygen species. In vivo studies demonstrate that the ICG component facilitates nIR fluorescence imaging that demonstrates accumulation of IV-administered nanoparticles in tumours. In vivo studies also demonstrate ultrasound- and light-mediated inhibition of tumor growth in animals treated with the platform. Since the platform consists entirely of clinically-approved agents it could find use in sonodynamic- and photodynamic-based therapies for cancer.
\end{abstract}

Key words: nanoparticle, sonodynamic, photodynamic, cancer, real-time, imaging, therapy 


\section{Introduction}

The search for non-invasive or minimally invasive approaches for the treatment of more deeply-seated cancers has led to the development of therapeutic regimes such as photodynamic therapy (PDT) [1]. The approach is based on the administration of a sensitiser that is taken up by tumours and is subsequently stimulated by light to produce cytotoxic reactive oxygen species (ROS). Although it delivers site-specific therapy, its acceptance in clinical practice as a mainstream cancer treatment modality is hindered, for the most part, by accumulation of sensitisers in skin [2] and the inability of light to penetrate deeply into living tissues [3] . The former necessitates the protection of patients from exposure to direct light and the latter precludes the treatment of inaccessible or more deeply-seated tumours. An alternative emerging approach involves the use of ultrasound to 'activate' the sensitising drug

and this is more generally known as sonodynamic therapy (SDT) [4]. Although many of the sensitisers used in PDT can also serve as sonosensitisers, the mechanism by which the sensitiser is activated by ultrasound in SDT is less clear. It is however generally accepted that site-specific toxicity results from the generation of ROS on exposure to ultrasound and a number of hypotheses have been suggested, all of which involve events that derive from ultrasound-induced cavitation [5,6]. This is supported by the observation that when sensitisers are chemically conjugated to the surface of microbubbles, the generation of ROS is enhanced in an acoustic field [7].

Since SDT uses ultrasound to activate the sensitiser, the major perceived clinical benefit is that it may be used to target more deeply-seated lesions since ultrasound can penetrate tissues more easily than light. Because SDT offers the potential to target more deeply seated lesions, accumulating sensitizer solely at the target site becomes essential in order to preclude uptake by non-target tissues and in particular by tissues that interspace the actual target and the ultrasound source. Achieving this would also preclude overall patient 
photosensitization; an adverse effect associated with many sensitisers employed in PDT as mentioned above. One potential means of accumulating sensitizer at the lesion would be to use a nanoparticle platform and exploit the enhanced permeation and retention (EPR) phenomenon exhibited by many solid tumors [8] Masters et al. have reviewed nanoparticlebased platforms for use in PDT and describe their ability to accumulate sensitizer in target lesions, although the inability of light to penetrate deeply into tissues in PDT remains a challenge [9]. Since tissue penetration is not a challenge for SDT, the use of a nanoparticlebased platform for more site-specific sensitizer delivery would provide significant advantage. Recently You et al. described the use of $\mathrm{TiO}_{2}$ nanoparticles in SDT demonstrating preferential uptake by tumours and therapeutic efficacy [10]. In addition to aiding the preferential accumulation of sensitiser in target lesions, nanoparticles can also be exploited to deliver multiple functionalities and this approach has been used to incorporate diagnostic as well as therapeutic capabilities in a single formulation. In another recent study, Chen et al. described the use mesoporous silica grown on reduced graphene nanosheet that was capped with rose bengal-conjugated to iron-oxide nanoparticles and demonstrated its use to simultaneously generate cytotoxic ROS and induce hyperthermia in an acoustic field [11]. Here the authors suggested that the graphene sheet provided a heat conducting base that induced hyperthermia and the iron nanoparticles provided seeding of cavitation to enhance ultrasound-enhanced ROS generation by rose bengal. The particles could also be used to magnetically target the construct to a specific locus and could provide a means of assessing accumulation at a target site using magnetic resonance imaging (MRI). Although a variety of novel and indeed extremely elegant nanoparticle-based platforms incorporating sensitisers and exhibiting multiple functionalities have recently been suggested for SDT, their potential behavioral characteristics in patients and their clinical acceptability remain unproven. 
Interestingly the first studies reporting the phenomenon of SDT exploited hematoporphyrin (HP) as the sensitizer [4]. Although its use as a sensitiser for SDT would be clinically acceptable, since it is a natural prophyrin and indeed derivatives of this are currently used clinically for PDT (Photofrin), its direct clinical exploitation is hindered by its limited solubility in aqueous media and its adverse biodistribution characteristics which result in prolonged skin photosensitization [12,13]. Since it has been suggested that nanotechnology could play a very significant role in the clinical exploitation of porphyrins [14], primarily because it could resolve issues associated with limited solubility and impact favorably on biodistribution, we decided to explore the possibility of using HP as a sensitiser payload in a nanoparticle-based platform. Poly(DL-lactic-co-glycolic acid) (PLGA) was used as a platform polymer because it is biocompatible, biodegradable and is approved by the United States Food and Drug Administration for use in humans [15]. In order to provide an imaging capability in the platform, it was decided to incorporate the cyanine dye, indocyanine green (ICG) into the HP-containing particles because it is a clinically-approved near infra-red (nIR) fluorescence imaging agent that is used to assess vascular patency [16]. Although ICG has also been reported to serve as a sensitizer that responds both to light and ultrasound, it was used in this study at concentrations that were at least 30 -fold lower than those required to elicit a therapeutic effect [17]. Here, we describe the physicochemical characterization of this stimulus-responsive platform. Using in vitro and in vivo target systems, it is shown to exhibit cytotoxic activity in response to light, ultrasound and combinations of both. The diagnostic capability of the platform and its exploitation therapy design is also described. The potential benefits offered by such a system in the treatment of solid tumours is discussed. 


\section{Materials and Methods}

\subsection{Preparation and characterization of nanoparticles}

$50 \mathrm{mg}$ of hematoporphyrin dichloride (HP) (>75\%; Sigma Aldrich, UK) and $10 \mathrm{mg}$ of indocyanine green (ICG) (Sigma Aldrich, UK) were dissolved in $3 \mathrm{~mL}$ of ethanol. Where HP containing nanoparticles were produced in the absence of ICG, the latter was omitted from this solution. A $100 \mathrm{mg}$ quantity of poly(DL-lactic-co-glycolic acid) (75:25; molecular weight: 66,000 - 107,000; Sigma Aldrich, UK) was dissolved in $4 \mathrm{~mL}$ of acetone. Following dissolution, both solutions were mixed and added dropwise to $40 \mathrm{~mL}$ of a vigorously-stirred 1 $\%(\mathrm{w} / \mathrm{v}$ ) aqueous solution of polyvinyl alcohol (MW 30,000 - 70,000; $87-90 \%$ hydrolyzed; Sigma Aldrich, UK) over a 7 min period. To enhance emulsification an ultrasound probe (6 mm; $20 \mathrm{kHz}$,Vibra-Cell; Sonics and Materials, Newton, CT, USA) was immersed in the polyvinyl alcohol solution and operated at 91 Watts (70\% of net power output) during addition of the PLGA mixture. The suspension was then stirred for $3 \mathrm{~h}$ and nanoparticles were recovered and washed once in distilled water, then in phosphate buffered saline (PBS) and finally in distilled water by centrifugation at $15,000 \mathrm{~g}$ for $30 \mathrm{~min}$. The pellet was then suspended in distilled water and centrifuged at $500 \mathrm{~g}$ for 3 min to remove larger aggregates before finally lyophilizing and storage at $-20^{\circ} \mathrm{C}$. To determine the HP and ICG content, 1 mg of nanoparticles was dissolved in acetone and the concentration of HP and ICG was determined using UV/Vis absorption spectrophotometry at 500nm and 800nm, respectively. Encapsulation efficiency is expressed as the mass of each payload recovered in the nanoparticle preparation as a \% the mass of each payload initially employed in the preparation. UV/Vis absorbance scans of nanoparticle preparations were performed using dilutions of a $1 \mathrm{mg} / \mathrm{mL}$ suspension of each nanoparticle preparation in PBS. In order to examine passive release of payload from the nanoparticles preparations, $1 \mathrm{~mL}$ of a $1 \mathrm{mg} / \mathrm{mL}$ suspension in PBS was placed inside dialysis tubing (SpectroPor, MW cut-off: 50,000, 
Spectrum Laboratories, Breda, The Netherlands) and this was then placed in a beaker containing $25 \mathrm{~mL}$ of distilled water. The solution outside the tubing was stirred using a magnetic stirrer and samples were harvested at the indicated times. HP and ICG that diffused into the external solution were determined by measuring the absorbance at $500 \mathrm{~nm}$ and $800 \mathrm{~nm}$, respectively.

\subsection{Determination of nanoparticle size}

Dynamic light scattering was employed to determine the diameter of the nanoparticles by analyzing dilutions of a $1 \mathrm{mg} / \mathrm{mL}$ suspension of particles in PBS using a Malvern Zetasizer Nano Z system (Malvern Instruments, Worcestershire, UK) and Zetasizer software version 6.12. The diameter of the nanoparticles was also determined using scanning electron microscopy by initially drying a sample of particles onto aluminum stubs, sputter coating with gold/palladium for $3 \mathrm{~min}$ at $18 \mathrm{~mA}$ and subsequently visualizing under high vacuum mode using a Quanta Environmental scanning electron microscope (FEI, Hillsboro, OR, USA).

\subsection{Determination of ROS production}

Ultrasound- and light-mediated ROS generation was determined using oxidation of 1,3diphenylisobenzofuran (DPBF) as described previously [6]. Essentially a nanoparticle preparation $(1 \mathrm{mg} / \mathrm{mL})$ was added to a $10 \mu \mathrm{M}$ solution of DPBF prepared in an ethanol:water (50:50) mixture, aerated for $10 \mathrm{~min}$ and exposed to ultrasound at the indicated power densities (expressed as spatial average, temporal peak) for $60 \mathrm{~min}$. A Sonidel SP100 sonoporator (Sonidel Ltd., Dublin, Ireland) was used in these studies and the instrument emitted ultrasound at a frequency of $1 \mathrm{MHz}$. During exposures, a 50\% duty cycle (pulse repetition rate $=100 \mathrm{~Hz}$ ) was employed. For treatment with light a Fenix LOD white light

emitting diode $\left(700 \mathrm{~mW} / \mathrm{cm}^{2}\right)$ (Fenix, Denver, USA) was used. Samples were harvested and 
scanned using UV/Vis spectrophotometry and ROS generation was determined by a reduction in absorbance at $410 \mathrm{~nm}$ relative to untreated control samples. Because of the photosensitive nature of the NP preparation, exposure to light was minimized during the studies.

\subsection{Mammalian cell culture and treatment in vitro}

RIF-1 cells were maintained in RPMI 1640 medium supplemented with glutamine (GlutaMAX, Invitrogen, UK) and 10\% (v/v) fetal bovine serum in a humidified atmosphere containing 5\% $\mathrm{CO} 2$ at $37^{\circ} \mathrm{C}$ as described previously [17]. Cells were dispensed into the wells of a 96-well plate at a concentration of $2 \times 10^{4}$ cells/well in serum-containing medium and incubated overnight prior to treatment. The medium was subsequently replaced by serum free medium and $20 \mu \mathrm{L}$ aliquots of a $1 \mathrm{mg} / \mathrm{mL}$ suspension of nanoparticles were dispensed into each well. $3 \mathrm{~h}$ later, medium was replaced with serum-containing medium and wells were treated with light, ultrasound or a combination of both. For treatment with light a Fenix LOD white light emitting diode $\left(700 \mathrm{~mW} / \mathrm{cm}^{2}\right)$ (Fenix, Denver, USA) was placed directly under each well to be treated. Cells were treated for $30 \mathrm{~s}$ and placed back in the incubator overnight. Cell viability was subsequently determined using an MTT-based cell viability assay and viability was expressed as a $\%$ of an untreated control population as described previously [17]. For ultrasound treatments, the transducer of a Sonidel SP100 sonoporator (Sonidel Ltd., Dublin, Ireland) was placed in direct contact with the bottom of each well to be treated. An ultrasound contact gel was employed to ensure contact with the bottom of each well and treatment consisted of exposing each well to ultrasound at a frequency of $1 \mathrm{MHz}$ for $30 \mathrm{~s}$ at a power density of $2.5 \mathrm{~W} / \mathrm{cm}^{2}$ (spatial average, temporal peak: SATP) and using a $50 \%$ duty cycle with a pulse repetition rate of $100 \mathrm{~Hz}$. Cells were again incubated overnight following treatment and cell viability was subsequently determined using the MTT-based cell viability assay. 


\subsection{Tumour treatment and nIR imaging}

Tumours were established by subdermal injection of 1 x $10^{6}$ RIF-1 cells in $100 \mu \mathrm{L}$ of PBS into the rear dorsum of host animals $(\mathrm{C} 3 \mathrm{H} / \mathrm{HeN}$ mice $)$ as described previously [17]. Animals were treated humanely and in accordance with licensed procedures under the UK Animals (Scientific Procedures) Act, 1986. When tumours had reached an average size of $250 \mathrm{~mm}^{3}$, animals were randomly distributed into groups for treatment with nanoparticles alone, nanoparticles plus light, nanoparticles plus ultrasound, nanoparticles plus light followed by ultrasound. In addition, controls without treatment and treated with light plus ultrasound in the absence of nanoparticles were employed. Animals were anaesthetized during all treatments using intraperitoneal administration of 1:2 dilutions of a 1:1 mixture of Hypnorm/Hypnovel. Where animals were treated with nanoparticles, $50 \mathrm{uL}$ aliquots of nanoparticles at a dose of $6.7 \mathrm{mg}$ nanoparticles/ $\mathrm{mL}$ of PBS were intravenously administered to each animal. Animals were then rested for $6 \mathrm{~h}$ before receiving treatment. For treatment with light, tumours were exposed to the Fenix LED emitting white light at a dose of 700 $\mathrm{mW} / \mathrm{cm}^{2}$ for $3 \mathrm{~min}$. For ultrasound treatments, contact between the transducer and the target tumour was maintained using an ultrasound contact gel and lesions were treated for 3.5 min. using ultrasound at a frequency of $1 \mathrm{MHz}$ and a power density of $3.5 \mathrm{~W} / \mathrm{cm}^{2}$ (SATP) using a $50 \%$ duty cycle at a pulse frequency of $100 \mathrm{~Hz}$. In cases where animals were treated with light and then ultrasound, the ultrasound was applied 15 min after treatment with light. Tumour volume was determined at the indicated times using $4\left(\pi r^{3}\right) / 3$, where $r$ is the radius obtained from measuring the geometric mean diameter using digital callipers. Data are expressed as a $\%$ of the starting tumour volume. For nIR fluorescence imaging, animals were anaesthetized using intraperitoneal administration of Hypnorm/Hypnovel and $50 \mu \mathrm{L}$ nanoparticles were administered intravenously at a dose of $6.7 \mathrm{mg} / \mathrm{mL}$ in PBS. Following administration, animals were placed in the chamber of a Xenogen IVIS ${ }^{\circledR}$ Lumina imaging 
system on fluorescence mode using the ICG filter set (excitation: 705-780 nm; emission: 810$885 \mathrm{~nm})$. Data were captured, analysed using the Living Image ${ }^{\circledR}$ software package version 2.60 and presented as arbitrary fluorescence units using the fluorescence efficiency mode to eliminate illumination intensity effects. For ex vivo imaging to examine the tissue distribution of nanoparticles, animals were sacrificed at $24 \mathrm{~h}$ and organs were surgically excised. Organs were then placed in the imaging system and fluorescence data were captured as described above.

\subsection{Statistical analyses}

Unless otherwise stated $n=3$ in experiments. Statistical analysis of significance was performed using analysis of variance and comparison of data groups was performed using the Tukey MCT in GraphPad Prism version 4. 


\section{Results}

\subsection{Characterisation of nanoparticles}

After preparation of the nanoparticles co-harboring HP and ICG using the solvent dispersion approach, suspensions were analysed using dynamic light scattering and it was found that particles had an average diameter of $200-270 \mathrm{~nm}$ with polydispersity indices (PDI) ranging from 0.118 to 0.320 . Data from a typical DLS run are shown in Fig.1S. These data were confirmed using scanning electron microscopy as shown in Fig.1A.

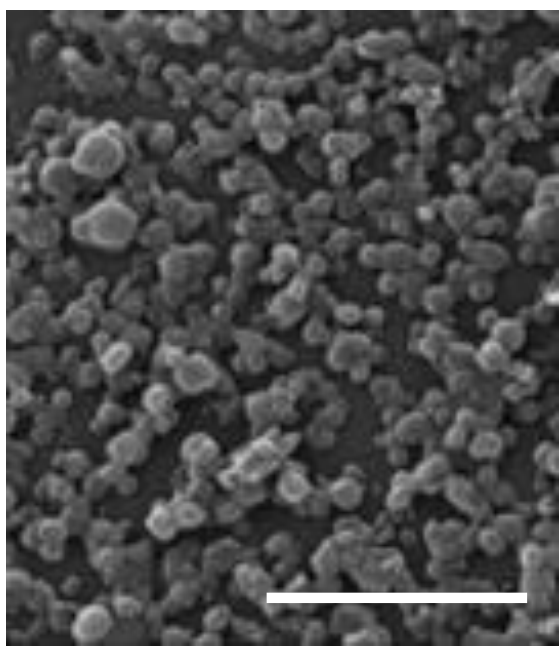

A

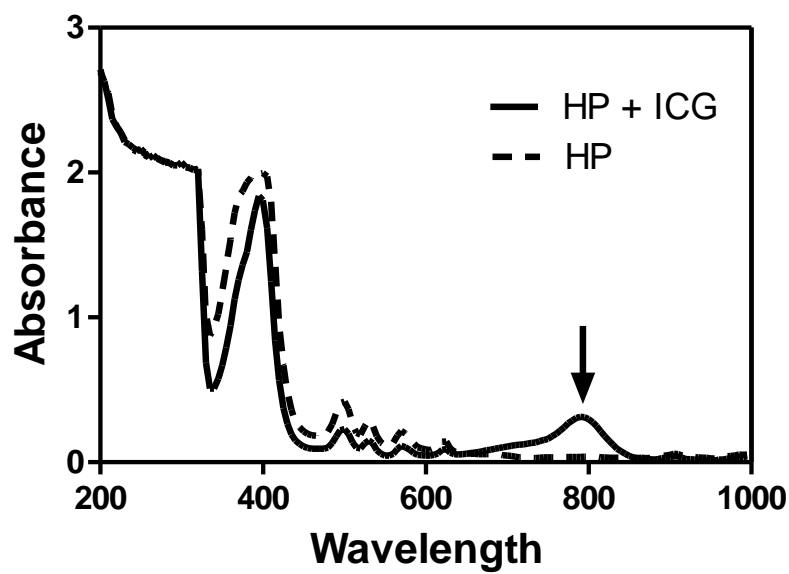

B

Fig. 1 (A) Scanning electron micrograph of nanoparticles (scale bar $=2 \mu \mathrm{m}$ ). The scale bar represents $2 \mu \mathrm{m} . \quad$ (B) Spectrophotometric scans of nanoparticles containing HP and HP together with ICG.

To confirm the presence of HP and ICG in the nanoparticle preparation, spectrophotometric scans of nanoparticles containing HP alone and HP together with ICG were obtained. The scan from the nanoparticles containing HP and ICG exhibited the characteristic absorption profile of HP together with an absorption peak characteristic of ICG 
in the $800 \mathrm{~nm}$ region of the spectrum (indicated by the arrow in Fig.1b). Spectrophotometric analysis following nanoparticle solvent extraction indicated that $1 \mathrm{mg}$ of nanoparticles contained $0.7 \mathrm{mg}$ of $\mathrm{HP}$ and $40 \mu \mathrm{g}$ ICG. The high proportion of HP in the vehicle probably results from the limited solubility of HP in aqueous media. From a 38\% yield, these data indicated a $70 \%$ encapsulation efficiency for HP and 24\% encapsulation efficiency for ICG.

In order to examine passive release of both HP and ICG from the nanoparticles, the preparation was incubated in PBS and the amount of HP and ICG leaking from the particles was determined by spectrophotometric analysis. Results indicate that whilst the majority of HP $(>70 \%)$ was released from the particles within a 40h period, a much lower percentage of the ICG was detected in the medium over this period (Fig.2A). Since it had been demonstrated in numerous studies that the photodynamic effect can be exploited to generate

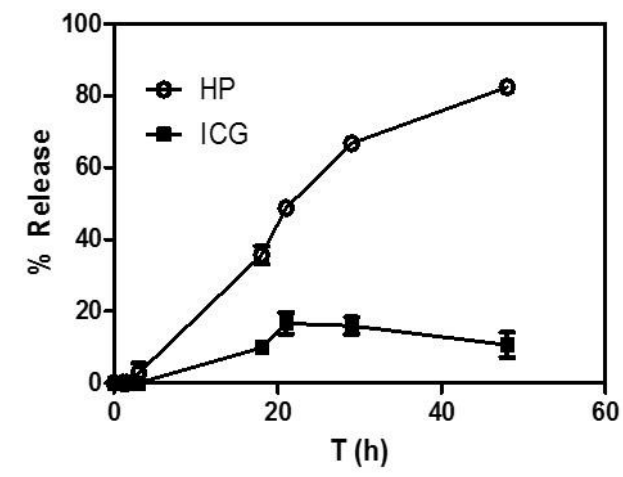

A

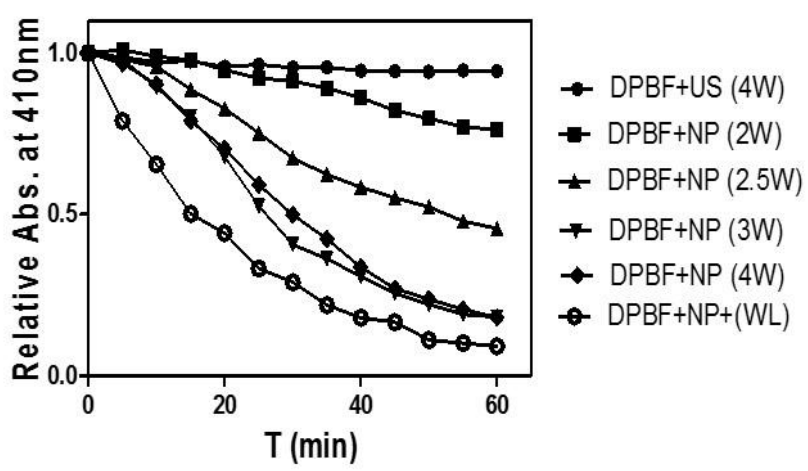

B

Fig.2 (A) Release of payloads HP and ICG from nanoparticles and (B) ROS production using $D P B F$ as a trap during exposure of nanoparticles (NP) to white light (WL) and ultrasound power densities ranging from $2-4 \mathrm{Wcm}^{-2}$. Error bars in (A) represent $\pm S D$ where $n=3$ and in panel (B) error bars are omitted in the interests of clarity with data points representing the averages where $n=3$. 
ROS from HP, it was decided to determine whether or not the HP-containing nanoparticles would mediate the generation of ROS in an acoustic field. To this end, nanoparticles were exposed to white light and an acoustic field at a variety of ultrasound power densities. The generation of ROS was determined using DPBF as a ROS trap as described previously [6] The data (Fig.2B) demonstrate that in the presence of either white light or ultrasound, ROS were generated in a time-dependent and ultrasound dose-dependent manner.

\subsection{In vitro studies using the nanoparticle platform}

Although nanoparticles provide a potential advantage in targeting agents to tumour tissues using the EPR effect, they can also provide benefit in aiding the cellular uptake of therapeutic agents [8]. In PDT or SDT, intracellular accumulation would provide advantage because internal organelles such as mitochondria serve as targets for ROS generated by those approaches [17]. Since the platform co-harbors ICG it was decided to determine whether or not this could be exploited together with nIR fluorescence imaging to assess cellular uptake of the platform by target cells in vitro. To this end a mouse radiation-induced fibrosarcoma cell line (RIF-1) was employed as the target. This cell line is used routinely in our laboratories as an in vitro target because it can also be used from a translational perspective to develop tumours in syngeneic host mice to provide in vivo targets [17]. Following incubation of the nanoparticles $(5 \mu \mathrm{l}$ of a $1 \mathrm{mg} / \mathrm{mL}$ suspension of nanoparticles in PBS) with target cells in 96- well plates, medium was replaced at the indicated times and plates were then examined for particle retention by cells using nIR fluorescence imaging. The results are shown in Fig. 3A with the inset providing a typical result obtained from fluorescence imaging. They demonstrate that particles are taken up by the cell population and uptake reaches saturation within $3-4 \mathrm{~h}$.

As shown in Fig.2B, the nanoparticles are capable of generating ROS in an acoustic field. In order to demonstrate that the nanoparticle platform could provide stimulus- 
responsive cytotoxicity, nanoparticles were incubated with the RIF-1 cells for $3 \mathrm{~h}$ to facilitate cellular uptake. Cell populations were then treated with white light, ultrasound and a combination of both. The effects of each treatment on cell viability are shown in Fig. 3B and demonstrate that when the nanoparticles are combined with light, ultrasound or light followed by treatment with ultrasound, a significant decrease $(\mathrm{p}<0.01)$ in cell viability was observed. While no statistically-significant difference was observed between nanoparticle-treated cells in the presence of either light or ultrasound, a significant decrease in viability

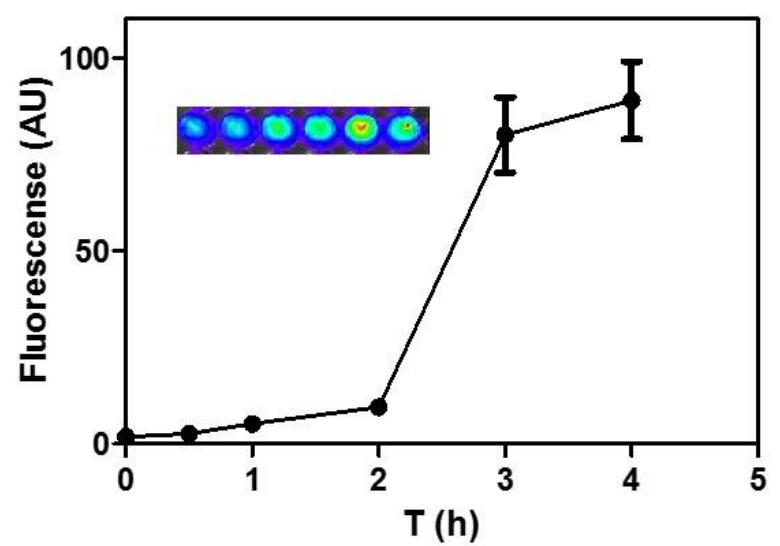

A

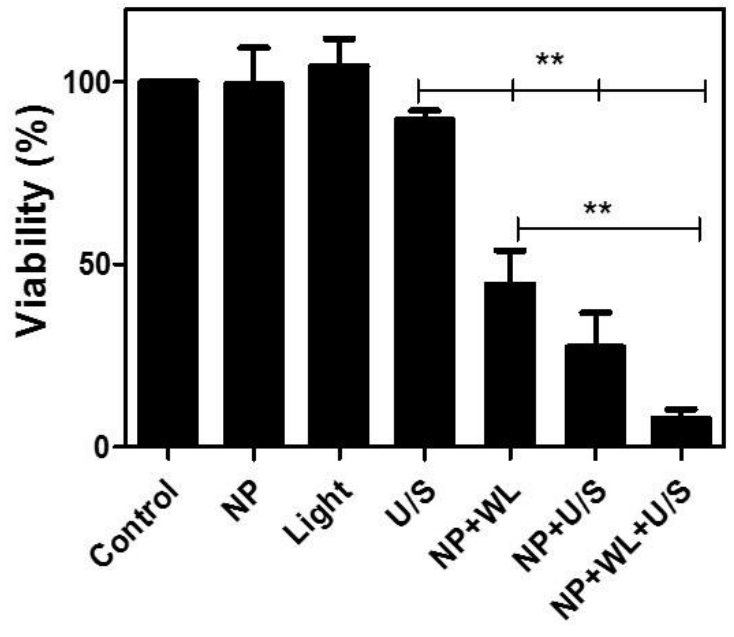

B

Fig.3 (A) nIR fluorescence imaging to detect uptake of nanoparticles by RIF-1 tumour cells. Fluorescence is expressed as counts detected from each well and the inset shows a typical result from fluorescence imaging with increasing incubation time from left to right. (B) The effects of light, ultrasound and light plus ultrasound on the RIF-1 cell viability in the presence and absence of NPs and bars represent \pm the standard error. $* * p<0.01$ was noted when the viability of cells treated with either stimulus alone was compared with cells treated with the combined stimuli. It was interesting to note that when cells were 
treated with either nanoparticles alone or the combined stimuli in the absence of nanoparticles no significant effect on cell viability was detected and this will be discussed below.

\subsection{In vivo studies using the nanoparticle platform}

Having established that the ICG payload could be used as a nIR fluorescence imaging probe (Fig.3A), it was decided to determine if this aspect could be used to monitor the distribution or fate of nanoparticles in vivo. To this end, a preparation of nanoparticles consisting of 0.23 mg (with respect to $\mathrm{HP}$ ) in a $50 \mu \mathrm{L}$ injection volume (PBS) was administered intravenously to tumor-bearing mice. Whole-body nIR fluorescence imaging was performed and a strong signal was immediately evident at the tumour (Fig.4A, left inset). The imaging

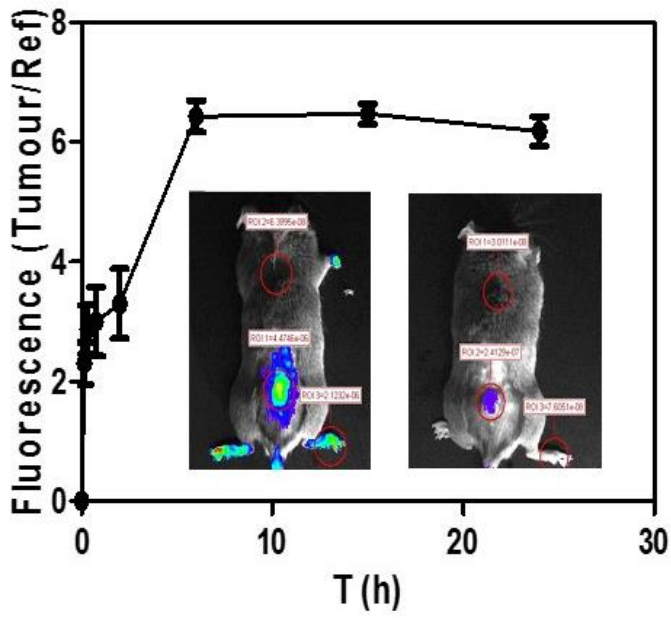

A

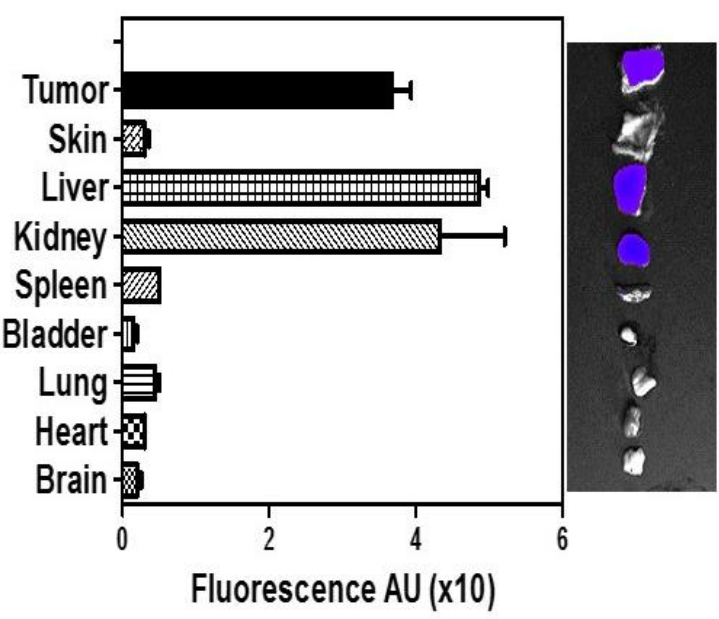

B

Fig.4 (A) nIR fluorescence imaging signal from tumour and peripheral tissues. Left insert is an image captured immediately after injection of nanoparticles and the right insert is an image captured at $24 \mathrm{~h}$. Regions of interest are represented by circles and data are presented as the ratio of the signal obtained from the tumour relative to that obtained from peripheral tissue. (B) nIR fluorescence imaging of surgically harvested tissues. 
system also detected a fluorescent signal from peripheral tissues as noted from the hind leg shortly after injection (Fig.4A, left inset) and this was used as a surrogate for more widespread circulatory distribution of the nanoparticles. Monitoring signals from both the tumour and peripheral tissues demonstrated a rapid increase in fluorescence in the tumour followed by a dramatic overall decline within the first 6h post administration (Fig.2S). Over this period however it was noted that the signal from the peripheral tissues decreased more rapidly and approximately $90 \%$ had been removed by $6 \mathrm{~h}$. Even after $24 \mathrm{~h}$, the signal from the tumour was still visible (Fig.4A, right inset) and when this was quantitatively expressed as a ratio to the signal obtained from the peripheral tissues, the data indicated a 6fold accumulation of nanoparticles in the tumour within $6 \mathrm{~h}$ and this ratio remained constant up to $24 \mathrm{~h}$. In order to confirm that nanoparticless were being accumulated in the tumour and to ascertain their tissue distribution, it was decided to image tissues that were surgically harvested from animals that were sacrificed $24 \mathrm{~h}$ after administration of nanoparticles. The data obtained are shown in Fig. 4B and they demonstrate that while the nanoparticle load in the tumour is significantly higher than in most tissues analyzed, significant fluorescence signals were found in both the liver and kidney. Data in Fig. 2A suggest that at $24 \mathrm{~h}, 16 \%$ of the ICG leaks from the nanoparticles and it is possible that some of the signal obtained in the kidney may result from leakage, nanoparticle degradation or a combination of both and may not completely reflect the presence of intact nanoparticles. Nevertheless, an extremely low signal was observed in the skin and this will be discussed further below.

In order to demonstrate therapeutic efficacy using multiple stimuli, nanoparticles were administered intravenously to tumour-bearing animals at the dose described above. Approximately $6 \mathrm{~h}$ after IV administration of the nanoparticles, tumours were treated with ultrasound, white light or a combination of both. In these studies, longer exposure times to both stimuli were chosen because of anticipated tissue attenuation that would not have 
existed in the in vitro studies. The data obtained are shown in Fig. 5 and they clearly demonstrate that while administration of either the nanoparticles or the stimuli had no impact on tumour growth, treatment using the nanoparticles together with each stimulus alone or in combination, resulted in a dramatic reduction in tumour growth. Indeed, even at day 9 , tumours had failed to reach their initial pre-treatment size. In addition, for animals treated with stimuli together with nanoparticles, no obvious damage to skin was observed confirming the observation above that the skin of these animals was not sensitised to the stimuli.

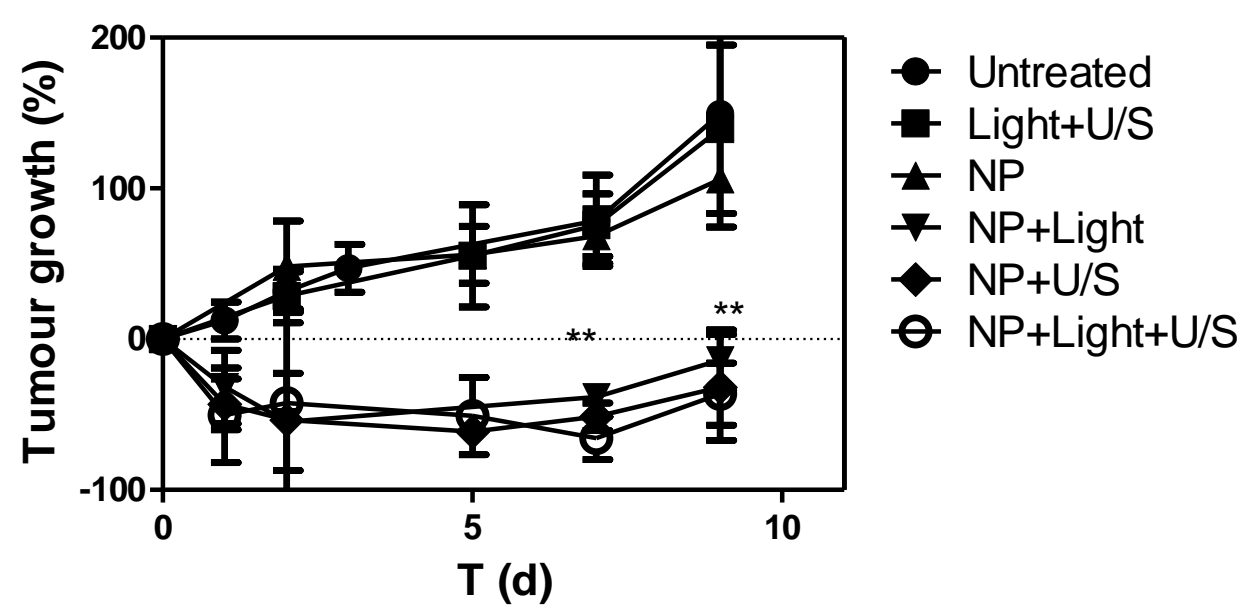

Fig.5 The effects of light, ultrasound (U/S) and light plus ultrasound on RIF-1 tumour growth in the presence and absence of intravenously-administered nanoparticles (NP). For the untreated control, tumours treated with light and ultrasound alone and those treated with NP alone, $n=3$ animals per group. For all other groups $n=4$. ** $p<0.01$ when groups treated with nanoparticles plus stimuli are compared with those treated with either nanoparticles alone or stimuli alone on the indicated days. 
Although the in vitro studies (Fig.3B) demonstrated that combining the stimuli resulted in a significant reduction in cell viability when compared with the effects obtained with each stimulus alone, no statistically significant decrease in tumour growth was detected in vivo when lesions were treated with the combined stimuli.

\section{Discussion}

As outlined above, SDT offers significant advantage in providing site-specific therapy for more deeply-seated cancers. However, in exploiting a highly tissue permeable stimulus such as ultrasound, it would be preferable if the sensitizer exhibited a significant degree of tumour specificity in order to preclude off-target effects, particularly in tissues lying between the target and the external ultrasound source. In order to explore the potential benefits afforded by a nanoparticle-based platform in delivering sensitizer for SDT, it was decided to design a platform that consisted entirely of clinically-acceptable components and to this end, nanoparticles consisting of clinically-approved PLGA containing the endogenous porphyrin, HP and the clinically-exploited nIR imaging agent ICG were prepared. The procedure chosen provided spherical nanoparticles with an average diameter of $270 \mathrm{~nm}$ and they compared favorably with those obtained using a similar method to prepare PLGA-based nanoparticles containing zinc (II) phthalocyanine for use in PDT [18]. While the encapsulation efficiency for HP was high, that for ICG was relatively low although it was noted that Saxena et al. reported lower encapsulation efficiencies in preparing ICGcontaining PLGA nanoparticles when similar ratios of ICG to PLGA were employed [19]. We are unaware of any study describing the preparation of HP-loaded PLGA nanoparticles, although a previous study has described encapsulation of HP in PLGA microbubbles 
(approximate dia. $2 \mu \mathrm{m}$ ) and in that study the encapsulation efficiency was 64\% [20] which approximates the $70 \%$ encapsulation efficiency reported here. The high levels of association between HP and PLGA in both studies may result from the limited solubility of HP in aqueous media which limits is use in either PDT or SDT. Despite this, it was noted that the HP did leak from the nanoparticles with over $70 \%$ released over a $40 \mathrm{~h}$ period and this was similar to the rate of release from the PLGA microbubbles described above [20]. This could provide advantage because once particles become trapped in tumour tissues, the passively released HP would be available to sensitise the surrounding tissues and this would complement cellular uptake of nanoparticles (Fig.3A). This aspect will be discussed further below. Conversely, the inability of the particles to release significant quantities of ICG could fortuitously be beneficial from an imaging perspective because the presence of a nIR fluorescent signal would reflect the presence of intact particles and not the free dye. Indeed, the fluorescence imaging capability of the nanoparticles was exploited here to demonstrate uptake by cell populations in vitro and it was found that the uptake saturation kinetics were similar to those reported in the literature for PLGA nanoparticles of the same shape and size $[21,22]$.

Having demonstrated that the nanoparticle preparation could generate ROS in the presence of an ultrasonic field at power densities well below those employed for hyperthermal tissue ablation [23], it was also demonstrated that the platform could deliver cytotoxic effects following exposure to ultrasound, light and light followed by ultrasound (Fig.3B). We believe that much of the toxicity delivered from the nanoparticles following exposure to stimuli results from stimulus-dependent generation of ROS from the HP component since the ICG was present in the particles at concentrations that were 30 -fold lower than those required to elicit a cytotoxic effect [17]. In these studies, it was also found that the stimuli or nanoparticles had no effect on cell viability when used alone and this is a 
very significant finding from a therapeutic perspective. In exploiting the EPR effect with nanoparticles to target solid tumors it is now generally accepted that some form of molecular targeting capability must be incorporated into the platform in order to minimize off-target effects [24]. This is certainly the case when the payload is a cytotoxic agent because PLGA nanoparticles tend to accumulate in the liver and this could have negative consequences for the patient [25]. In the current study, the payload and stimuli are non-toxic and only elicit toxicity when both are combined. This highlights one of the major benefits of the platform since it precludes the necessity for sophisticated molecular targeting strategies.

In exploring the behavior of the platform in mice bearing syngeneic tumours a dose of $0.23 \mathrm{mg}$ (with respect to $\mathrm{HP}$ ) in a $50 \mu \mathrm{L}$ injection volume was administered intravenously to tumor-bearing mice. Based on an animal weight of $30 \mathrm{~g} / \mathrm{mouse}$, this dose represented approximately 4 times the dose of Photofrin ${ }^{\circledR}$ recommended by the United States FDA for PDT-based treatment of esophageal cancer, endobronchial cancer and high-grade dysplasia in Barrett's Esophagus ( $2 \mathrm{mg} / \mathrm{kg}$ ) [26]. The benefit afforded by inclusion of the HP in the nanoparticle platform is evidenced by the observation that it would not have been possible to inject this amount of HP intravenously in PBS because of its limited solubility in aqueous media. Using whole body fluorescence imaging following administration of the platform it was possible to detect a strong fluorescence signal from tumours and peripheral tissues. Over time, the signals reduced in intensity although that in the tumours reduced more slowly (Fig.2S and Fig.4). Although semi-quantitative, this level of analysis demonstrated that the imaging capability provided by the ICG in the nanoparticles permitted direct, real-time visualisation of the appearance and retention of those nanoparticles in tumour tissues as well as monitoring the disappearance of material from peripheral or non-target tissues. The ability to observe these distributions in real time in the clinic would provide the practical benefit of being able to directly identify the appropriate time to administer the stimulus and 
this would preclude damage to off-target tissues. Subsequent fluorescence imaging analysis of tissues harvested at $24 \mathrm{~h}$ indicated significant signals from the tumour, liver and kidney with extremely low levels of fluorescence in all other tissues examine. Although signals in the liver and kidney were high, this is a significant improvement on the distribution of hematoporphyrin derivative (a chemically-derivatised form of hematoporphyrin) following administration in its free form where 4-fold that found in the tumour was detected in the liver at $24 \mathrm{~h}$ post injection into mice [27] The latter study also demonstrated accumulation of porphrin in the kidney that was 2.5 -fold that in the tumour and levels in the skin were similar to that found in the tumour. In our studies imaging of skin samples yielded a very low signal, indicating poor retention of nanoparticles in the skin and this would imply that the skin was not sensitised. This could represent an additional benefit of the nanoparticle platform described here since one well-known side effect associated with porphyrin-based PDT is hypersensitivity of the skin to ambient light. Despite the relatively qualitative nature of our analysis, the pattern of nanoparticle tissue distribution obtained from direct nIR fluorescence imaging exhibits striking similarities to those described in previous studies where more quantitative methods were employed to detect the distribution of PLGA nanoparticles in tissues [28]. Using PLGA nanoparticles harboring cisplatin, Alan et al. had shown significant accumulation of nanoparticle payload (cisplatin) in liver, kidney and spleen [25]. Using ${ }^{99 \mathrm{~m}} \mathrm{Tc}$-labelled PLGA nanoparticles of similar size to those used in the current study and imaging with a gamma camera, Souza et al., demonstrated significant accumulation of nanoparticles in liver, kidney and spleen $8 \mathrm{~h}$ after administration [29]. As mentioned above, since the approach described in the current study employs payloads that are non-toxic in the absence of stimulus, the impact of accumulation in organs such as the liver and kidney would be of less clinical significance than a similar degree of accumulation of nanoparticles harboring a toxic chemotherapeutic drug. 
When tumours in animals treated with nanoparticles were exposed to light or ultrasound, similar reductions in tumour size were observed and this was in agreement with the data obtained during the in vitro studies. These data confirm the observations from the in vitro studies and demonstrate that the SDT approach is as good as the clinically-accepted PDT-based approach using this nanoparticulate platform. It was also noted in these studies that where nanoparticles and stimuli were used, no effects were observed on the skin overlying the tumours and this corroborated the imaging results which suggested that the skin did not contain any nanoparticles. In addition, neither the nanoparticles nor the stimuli, when used separately, had any significant effect on tumour growth and this again emphasizes the benefit afforded by this stimulus-responsive platform. Interestingly, no significant enhanced effect on tumour size was observed when both stimuli were combined as observed during the in vitro studies. Indeed, a number of studies have demonstrated that combining ultrasound and light, results in better effects in vitro and in vivo using other sensitisers $[4,6,30]$. However, since translation of observations from in vitro to in vivo is somewhat challenging, particularly with ultrasound because of the difficulty associated with mimicking true in vivo exposure conditions, observing benefit afforded by the combined stimuli will necessitate further optimisation. This could potentially involve a more detailed examination of the stimulus levels applied in vivo. Nevertheless, despite the lack of an observed benefit afforded using the combined stimuli, the system could offer potential benefit in situations where PDT may be of limited use in the treatment of less accessible lesions. As mentioned, one of the major benefits afforded by SDT is the ability of the stimulus to penetrate deeply into tissues. In a recent study comparing SDT with PDT for the treatment of skin cancer (both melanoma and non-melanoma skin cancers) we have demonstrated that senstisers may be activated at depth using ultrasound as the stimulus [31]. In addition, it was found that ultrasound could also elicit a therapeutic effect in melanotic lesions where the presence of 
melanin compromised the efficacy of PDT. Since the current nanoparticulate platform can respond to both light and ultrasound, it could be activated with ultrasound to yield a therapeutic response when the use of light would be contraindicated.

In comparing the existing platform with other nanoparticle-based sensitisers for use in SDT, a recent review on the use of nanoparticles in SDT suggested that one of the limitations of singlet oxygen-generating nanoparticles is the short lifetime of that species $(<3.5 \mu \mathrm{s})$ which limits its diffusion distance thereby limiting therapeutic efficacy [32]. This would present a particular challenge where the actual nanoparticle itself is the sensitizer or where the sensitizer is covalently attached to the nanoparticle where stimulus-generated ROS would be expected to diffuse away from the actual sensitizer in order to elicit a cytotoxic effect $[10,11,32,33]$. Such a limitation would also be exacerbated in less vascularized tumours and those comprising a high proportion of stroma, both of which are negative prognostic markers in cancer as a result of limited diffusion and dispersion of therapeutics throughout the tumour tissues. Clearly, this is not the case with the nanoparticles described here and therapeutic efficacy may result, at least in part, from the ability of the HP to diffuse from the particles (Fig. 3A) and more effectively sensitise cells at the target site. In addition, because the nanoparticles are composed of a biodegradable polymer, degradation of the nanoparticles in the tumour would enhance release and dispersion of HP so that it could be taken up more effectively by tumour cells, thereby further negating limitations associated with the short lifetimes of stimulus-generated ROS.

Although others have reported the use of nanoparticle-based platforms in PDT and more recently in SDT $[10,11,32,33]$ none have described a single multifunctional, biodegradable nanoparticle-based preparation with diagnostic capabilities, composed of clinically-approved reagents for use in both SDT and PDT and enabling the slow release of sensitizer that could enhance dispersion of that sensitizer at the target site. Use of the PLGA 
nanoparticle platform enables the use of therapeutic doses of an otherwise insoluble porphyrin and provides a degree of tumour specificity that is superior to that obtained with clinically approved, porphyrin-based sensitisers. The particles are non-toxic in the absence of stimulus and when used together with harmless doses of light and/or ultrasound, provide site-directed toxicity. By using the imaging capability provided by ICG in the nanoparticles to monitor their uptake by target tissues and removal of nanoparticles from non-target tissues, we suggest that this aspect may be exploited to identify the appropriate time for stimulus administration in order to eradicate off-target effects. It is also worth noting that the platform is flexible enough to accommodate alternative imaging agents that could further enhance the imaging capabilities of the system. We suggest that this highly biocompatible and versatile nanoparticle-based platform provides a flexible, multifunctional formulation for use in photodynamic- and/or sonodynamic-based therapeutic regimes aimed at treating solid tumours.

\section{Acknowledgements}

This work was funded in part by a EUROSTARS award (Project No.E5650-UGen). CMcE acknowledges the Department of Employment and Learning (DEL), Northern Ireland for a $\mathrm{PhD}$ studentship.

\section{References}

1. T.J Dougherty, G.B.Grindley, R. Fiel, K.R.Weishaupt and D.Boyle, Photodynamic therapy II. Cure of animal tumors with hematoporphyrin and light. J. Natl. Cancer Inst. 55 (1975) 115-121.

2. T.J.Dougherty, C.J. Gomer, B.W.Henderson, G.Jori,„D. Kessel, M.Korbelik, J.Moan and Q. Peng, Photodynamic Therapy, J. Natl. Cancer Inst., 90 (1998) 889-905. 
3. J.R. Starkey, A.K. Rebane, M.A. Drobizhev, F. Meng, A.Gong, A. Elliott, K. McInnerney and C.W. Spangler, New two-photon activated photodynamic therapy sensitizers unduce xenograft tumor regressions after near-IR laser treatment through the body of the host mouse, Clin Cancer Res., 14 (2008) 6564-6573.

4. N. Yumita, R. Nishigaki, K. Umemura and S. Umemura, Hematoporphyrin as a sensitizer of cell-damaging effect of ultrasound. Jpn. J. Cancer Res., 80 (1989) 219222.

5. D. Costley, C. McEwan, C. Fowley, A.P. McHale, J. Atchison, N. Nomikou and J.F. Callan, Treating cancer with sonodynamic therapy: a review, Int. J. Hyperthermia., 31 (2015) 107-117.

6. B. McCaughan, C. Rouanet, C. Fowley, N. Nomikou, A.P. McHale, P.A. McCarron and J.F. Callan, Enhanced ROS production and cell death through combined photoand sono-activation of conventional photosensitizing drugs, Bioorg. Med. Chem. Lett., 21 (2011) 5750-5752.

7. N. Nomikou, C. Fowley, N.M. Byrne, B. McCaughan, A.P. McHale and J.F. Callan., Microbuble-sonosensitiser conjugates as therapeutics in sonodynamic therapy, Chem Commun (Camb)., 48 (2012) 8332-8334.

8. V. Torchilin, Tumor delivery of macromolecular drugs based on the EPR effect, Adv. Drug Deliv. Rev., 63 (2011) 131-135.

9. A. Master, M. Livingston and A. Sen Gupta, Photodynamic nanomedicine in the treatment of solid tumors: perspectives and challenges, J. Control. Release, 168, (2013) 88 .

10. D.G. You, V.G. Deepagan, W. Um, S. Jeon, S. Son, H. Chang, H.I. Yoon, Y.W. Cho, M. Swierczewska, S. Lee, M.G. Pomper, I.C. Kwon, K. Kim and H.J.Park., ROS- 
generating $\mathrm{TiO}_{2}$ nanoparticles for non-invasive sonodynamic therapy of cancer, Sci. Rep., 6 (2016) DOI: 10.1038/srep23200.

11. Y.W. Chen, T.Y. Liu, P.H. Chang, P.H. Hsu, H.L. Liu, H.C. Lin and S.Y. Chen., A theranostic nrGO@MSN-ION nanocarrier developed to enhance the combination effect of sonodynamic therapy and ultrasound hyperthermia for treating tumor, 8 (2016) 12648-12657.

12. D. Mew, V. Lum, C-K Wat, G.H.N. Towers, C-H.C. Sun, R.J. Walter, W. Wright, M.W. Berns and J.G.Levy, Ability of specific monoclonal antibodies and conventional antisera conjugated to hematoporphyrin to label and kill selected cell lines subsequent to light activation. Cancer Res., 45 (1985) 4380-4386.

13. J. Moan and Q. Peng, An outline of the history of PDT in: T. Patrice (Ed), Photodynamic Therapy, Royal Society of Chemistry, Cambridge UK, (2003) 1-18.

14. Y. Zhou, X. Liang, Z. Dia. Porphyrin-loaded nanoparticles for cancer theranostics, Nanoscale, 8 (2016) 12394-12405.

15. M.S. Shive and J.M. Anderson, Biodegradation and biocompatibility of PLA and PLGA microspheres, Adv. Drug Deliv. Rev., 28 (1997) 5-24.

16. X. Zheng, D. Xing, F. Zhou, B. Wu and W.R. Chen, Indocyanine green-containing nanostructure as a near infrared dual-functional targeting probes for optical imaging and photothermal therapy, Mol Pharm., 8 (2011) 447-456.

17. N. Nomikou, C. Sterrett, C. Arthur, B. McCaughan, J.K. Callan and A.P. McHale, The effects of ultrasound and light on indocyanine-gree-treated tumour cells and tissues, ChemMedChem, 7 (2012) 1465-1471.

18. E. Ricci-Junior and J.M. Marchetti, Preparation, characterization, photocytotoxicity assay of PLGA nanoparticles containing zinc (II) phthalocyanine for photodynamic therapy use, J. Microencapsul., 23 (2006) 523-538. 
19. V, Saxena and M. Sadoqi, J. Shao, Indocyanine green-loaded biodegradable nanoparticles: preparation, physicochemical characterization and in vitro release. Int. J. Pharm., 278 (2004) 293-301.

20. Y. Zheng, Y. Zhang, M. Ao, P. Zhang, H. Zhang, P. Li, L. Qing, Z. Wang and H. Ran, Hematoporphyrin encapsulated PLGA microbubbe for contrast enhanced ultrasound imaging and sonodynamic therapy. J. Microencapsul., 29 (2012) 437-444.

21. L. Chronopoulou, A. Amalfitano, C. Palocci, G. Nocca, C. Calla and A. Arcovito, Dexamethasone-loaded biopolymeric nanoparticles promote gingival fibroblasts differentiation. Biotechnol Prog., 31 (2015) 1381-1387.

22. K.Y. Win and S.S. Feng, Biomaterials, Effects of particle size and surface coating on cellular uptake of polymeric nanoparticles for oral delivery of anticancer drugs, 26 (2005) 2713-2722.

23. Y, Zhou, L. Zhai, R. Simmons and P. Zhong, Measurement of high intensity focused ultrasound fields by a fiber optic probe hydrophone. J. Acoust. Soc. Am. 120 (2006) 676-685.

24. M. Nui, Y.W. Naguib, A.M. Aldayel, Y.C. Shi, S.D. Hursting, M.A. Hersh and Z. Cui. Biodistribution and in vivo activities of tumor-associated macrophage-targeting nanoparticles incorporated with doxorubicin. Mol. Pharm. 11 (2014) 4425-4436

25. N. Alam, V. Khare, R. Dubey, A. Saneja, M. Kushwaha, G. Singh, N. Sharma, B. Chandan and P.N. Gupta, Biodegradable polymeric system for cisplatin delivery: development, in vitro characterization and investigation of toxicity profile, Mater. Sci. Eng. C. Mater. Biol. Appl., 38 (2014) 85-93.

26. Photofrin Prescribing information FDA (United States of America), http://www.accessdata.fda.gov/drugsatfda_docs/label/2011/020451s0201bl.pdf accessed June, 2016. 
27. C.J. Gomer and T.J. Dougherty. Determination of $\left[{ }^{3} \mathrm{H}\right]$ and $\left[{ }^{14} \mathrm{C}\right]$ hematoporphyrin derivative distribution in malignant and normal tissue. Cancer Res., 39 (1979) 146151.

28. B. Semete, L. Booysen, Y. Lemmer, L. Kalombo, L. Katata, J. Vershcoor and H.S. Swai, In vivo evaluation of the biodistribution and safety of PLGA nanoparticles as drug delivery systems, Nanomedicine, 6 (2010) 662-671

29. A.C. Souza, A.L. Nascimento, N.M. de Vasconcelos, M.S. Jeronimo, I.M. Siqueira, L. R-Santos, D.O. Cintra, L.L. Fuscaldi, O.R. Pires Junior, R. Titze-de-Almeida, M.F. Borin, S.N. Bao, O.P. Martins, V. N. Cardoso, S.O. Fernandes, M.R. Mortari, A. C. Tedesco, A.C. Amaral, M.S. Felipe and A.L. Bocca, Activity and in vivo tracking of amphotericin B loaded PLGA nanoparticles, Eur. J. Med. Chem. 95 (2015) 267-176.

30. P. Wang, C. Li, X. Wang, W. Xiong, X. Feng, Q. Liu, A.W. Leung and C. Xu, Antimetastatic and pro-apoptotic effects elicited by combination photodynamic therapy with sonodynamic therapy on breast cancer both in vitro and in vivo, Ultrason. Sonochem. 23 (2015) 116-127.

31. C. McEwan, H. Nesbitt, D. Nicholas, O.N. Kavanagh, K. McKenna, P. Loan, I.G. Jack, A.P. McHale, J.F. Callan, Comparing the efficacy of photodynamic and sonodynamic therapy in non-melanoma and melanoma skin cancer. Bioorg. Med. Chem. 24 (2016) 3023-3028

32. H. Xu, X. Zhang, R. Han, P. Yang, H. Ma, Y. Song, Z. Lu, W. Yin, X. Wu and H. Wang, Nanoparticles in sonodynamic therapy: state of the art review, RCS Advances, 6 (2016) 50697-50705.

33. A. Sazgarnia, A. Shanei, N.T. Meibodi, H. Eshghi, H. Nassirli, Sonodynamic therapy. In vivo study on a colon tumor model. J. Ultrasound Med., 30 (2011) 13211329. 
